\section{Add radioactivity to the Listeria}

\section{By Michael J. Haas, Senior Writer}

An Albert Einstein College of Medicine of Yeshiva University team has used radiolabeled Listeria monocytogenes to efficiently treat metastatic pancreatic cancer in mice. ${ }^{1}$ Future studies could involve comparing or combining the strategy with existing nonradioactive immunotherapies that utilize the bacterium as a vector.

L. monocytogenes is a food-borne pathogen that can infect a range of mammalian cell types and cause potentially fatal illness (listeriosis) in immune-compromised individuals. For the last decade, attenuated strains of the bacterium have served as vectors for delivering antigens with high specificity to dendritic cells, thereby eliciting an immune response to cancer or infection. $^{2}$

At least two companies-Advaxis Inc. and

"It would also be interesting
to see a therapeutic that
harnessed all three ways in
which Listeria can be used
against cancer: direct killing
of tumor cells, delivery of
a therapeutic payload to
tumors and delivery of cancer
antigens to immune cells."
-Yvonne Paterson,
University of Pennsylvania

Dadachova, professor of radiology and of microbiology and immunology at Albert Einstein College of Medicine, proposed giving L. monocytogenes a radioactive payload.

In a mouse model for murine pancreatic cancer, an attenuated strain of L. monocytogenes replicated efficiently in metastatic tumors, poorly in primary tumors and not at all in normal tissues such as the spleen.

Next, the team linked an anti-Listeria antibody labeled with the radionuclide rhenium-188 $\left({ }^{188} \mathrm{Re}\right)$ to the surface of the attenuated L. monocytogenes. The team chose ${ }^{188} \mathrm{Re}$ because its half-life of about 17 hours allowed the delivery of a therapeutic dose of radiation in a relatively short time frame.

In the pancreatic cancer models, L. monocytogenes loaded with the largest quantity of ${ }^{188} \mathrm{Re}$-dubbed RL-200-decreased the number of metastases in liver and other organs by about $90 \%$, and the unlabeled bacterium decreased the number by about $50 \%$, compared with vehicle or ${ }^{188} \mathrm{Re}$ alone. In primary tumors, RL-200 and the unlabeled bacterium decreased growth by about $64 \%$ and $20 \%$, respectively.

Histopathological analyses showed that RL-200 did not cause damage to liver, kidneys or other normal tissues.

Data were reported in the Proceedings of the National Academy of Sciences.

Collectively, the results demonstrate that "radioactive Listeria could be valuable as an early second-line therapy-following removal of the primary pancreatic tumor-to treat
Aduro BioTech Inc. - have vaccines or immunotherapies based on attenuated $L$. monocytogenes in development to treat various cancers and infectious diseases.

Another advantage of the bacterium is that it survives longerand thus replicates to a greater extent-in the immunosuppressive environment of tumors than in normal tissues. This has allowed it to be used for cancer imaging in animal models ${ }^{3}$ and for expressing enzymes capable of converting chemotherapy prodrugs into active therapeutic agents in human breast cancer and mouse melanoma cell lines. ${ }^{4}$

Moreover, a 2009 study co-led by Claudia Gravekamp and Yvonne Paterson showed that an attenuated L. monocytogenes itself had efficacy in cancer. The bacterium decreased primary and metastatic tumor growth in mouse models for breast cancer almost as effectively as the same bacterial vector expressing a cancer antigen. ${ }^{5}$

Gravekamp is associate professor of microbiology and immunology at Albert Einstein College of Medicine. Paterson is professor of microbiology at the Perelman School of Medicine at the University of Pennsylvania, professor of nursing and associate dean for research at the University of Pennsylvania's School of Nursing and cofounder of Advaxis.

Those 2009 findings prompted a new team, co-led by Gravekamp and Ekaterina Dadachova, to start thinking about how to boost the tumoricidal activity of L. monocytogenes rather than using it as an antigen-delivery vehicle. existing metastases and prevent the development of new metastases," Gravekamp told SciBX.

She added that RL-200 should also be able to eliminate any remaining primary tumor cells in patients because those cells would be actively dividing. In contrast, the primary tumors in the mouse models-in which RL-200 replicated poorly-were dormant.

"Using Listeria to deliver a payload to tumors is a clever idea," Paterson said. In the 2009 study, "we looked at Listeria alone and showed its effects on tumor growth and the mechanism by which it kills tumors. But we know this isn't always very effective, maybe because not all cancer cells get infected or contain enough of the bacteria to kill them. Adding a radionuclide would help because uninfected tumor cells that surround the Listeria-infected cell could be killed by radiation."

\section{Radiotherapy, vaccine or both?}

It is not yet clear whether L. monocytogenes will have better efficacy as a vehicle for delivering radioactive payloads or as a vector for expressing cancer antigens in immune cells. Ultimately, it might be possible to combine both uses of the bacterium into one product.

Paterson said the two therapeutic approaches could be assessed with a "side-by-side comparison in the same tumor model. It would also be interesting to see a therapeutic that harnessed all three ways in which Listeria can be used against cancer: direct killing of tumor cells, delivery of a therapeutic payload to tumors and delivery of cancer antigens to 
immune cells."

Steven Bodovitz, Aduro's associate director for strategic development, said combining radiotherapy and immunotherapy could be effective, based on results the company saw in a Phase I trial of its CRS-207 (ANZ-207). The product is a vaccine based on a live, attenuated strain of L. monocytogenes that expresses human mesothelin.

In the trial, two of three patients with non-small cell lung cancer (NSCLC) received the vaccine and subsequently received local radiotherapy intended to be palliative. "Both subjects demonstrated a systemic antitumor response that indicated synergy between" the two types of therapy, he said.

Thus, although Aduro and the PNAS team took differing approaches to combining L. monocytogenes and radiotherapy in the treatment of cancer, "we agree that the combination of radiotherapy and immunotherapy has the potential to be synergistic," Bodovitz said.

CRS-207 also is in Phase II testing to treat pancreatic cancer and Phase I to treat malignant pleural mesothelioma. The company will present results from the trial this August at the annual meeting of the American Society of Clinical Oncology.

Aduro CSO Thomas Dubensky Jr. cautioned that combining immunotherapy and radiotherapy into one attenuated strain of $L$. monocytogenes and a cancer antigen, is in Phase I testing to treat liver cancer.

Aduro CSO Thomas Dubensky Jr. cautioned that combining immunotherapy and radiotherapy into one attenuated strain of L. monocytogenes might be challenging because different strains are not equally suited to both purposes.

For example, he said, "our attenuated strain of Listeria was designed to induce an immune response by targeting dendritic cells and other phagocytic cells of the immune system, not deliver a toxic payload directly to cancer cells."

Nevertheless, Gravekamp said it should be possible to develop a single Listeria-based therapeutic that delivered antigens to immune cells and a radioactive payload to tumor cells. "A chemotherapeutic payload would probably kill the immune cells it infects, but our radionuclide might not," she said.

She added that the team already has shown that RL-200 does not kill myeloid-derived suppressor cells-a type of immune cell involved in wound repair, inflammation and cancer.

However, she said, "we are definitely more interested in combination therapies that use RL-200 and a separate cancer vaccine because one type of therapy will not be sufficient against metastatic cancer."
It would also be important to establish that $L$. monocytogenes is safe when delivering a therapeutic payload, said Robert Fine, associate professor of medicine and director of experimental therapeutics at the Herbert Irving Comprehensive Cancer Center at Columbia University.

"Even though the bacterium is attenuated, it's still live and might be able to cause a dangerous, even fatal, infection," he said.

The use of L. monocytogenes as a vector in cancer vaccines does not raise the same concern because those bacteria do not survive long enough to replicate, he said.

Bodovitz agreed that additional studies are needed to establish the safety of the radioactive Listeria. He also wanted to see survival data for RL-200 in the animal models and know more about the agent's mechanism of action.

Additionally, Fine said RL-200 should be tested in models for metastatic pancreatic cancer-such as those expressing mutant $\mathrm{K}$-Ras and $p 53$ - that better correlate with human disease.

Gravekamp said the team will next test RL-200 in at least two xenograft models for metastatic pancreatic cancer.

Albert Einstein College of Medicine has patented the PNAS findings, and the IP is available for licensing. "We are very interested in finding an industry partner to take this approach into the clinic," said Gravekamp.

Haas, M.J. SciBX 6(17); doi:10.1038/scibx.2013.407

Published online May 2, 2013

\section{REFERENCES}

1. Quispe-Tintaya, W. et al. Proc. Natl. Acad. Sci. USA; published online April 22, 2013; doi:10.1073/pnas.1211287110 Contact: Claudia Gravekamp, Albert Einstein College of Medicine of Yeshiva University, Bronx, N.Y. e-mail: claudia.gravekamp@einstein.yu.edu

Contact: Ekaterina Dadachova, same affiliation as above e-mail: ekaterina.dadachova@einstein.yu.edu

2. Singh, R. \& Paterson, Y. Expert Rev. Vaccines 5, 541-552 (2006)

3. Yu, Y.A. et al. Nat. Biotechnol. 22, 313-320 (2004)

4. Stritzker, J. et al. BMC Cancer 8, 94; published online April 10, 2008; doi:10.1186/1471-2407-8-94

5. Kim, S.H. et al. Cancer Res. 69, 5860-5866 (2009)

COMPANIES AND INSTITUTIONS MENTIONED

Aduro BioTech Inc., Berkeley, Calif.

Advaxis Inc. (OTCBB:ADXS), North Brunswick, N.J.

Albert Einstein College of Medicine of Yeshiva University, Bronx, N.Y.

American Society of Clinical Oncology, Alexandria, Va.

Columbia University, New York, N.Y.

Perelman School of Medicine at the University of Pennsylvania, Philadelphia, $\mathrm{Pa}$.

University of Pennsylvania, Philadelphia, Pa. 